\title{
Improvement of Visuo-spatial Function Assessed by Raven's Colored Progressive Matrices in Dementia with Lewy Bodies by Donepezil Treatment
}

\author{
Yuta Yoshino ${ }^{1}$, Takaaki Mori ${ }^{1}$, Taku Yoshida ${ }^{1}$, Yasutaka Toyota ${ }^{2}$, Hideaki Shimizu ${ }^{1}$, Jun-ichi Iga ${ }^{1}$, \\ Shusaku Nishitani ${ }^{2}$, Shu-ichi Ueno ${ }^{1}$ \\ ${ }^{1}$ Department of Neuropsychiatry, Molecules and Function, Ehime University Graduate School of Medicine, Toon, ${ }^{2}$ Department of Neuropsychiatry, \\ Zaidan Niihama Hospital, Niihama, Japan
}

\begin{abstract}
Objective: Donepezil is used to improve cognitive impairment of dementia with Lewy bodies (DLB). Visuo-spatial dysfunction is a well-known symptom of DLB. Non-verbal Raven's Colored Progressive Matrices (RCPM) were used to assess both visual perception and reasoning ability in DLB subjects treated with donepezil.

Methods: Twenty-one DLB patients (mean age, $78.7 \pm 4.5$ years) were enrolled. RCPM assessment was performed at the time of starting donepezil and within one year after starting donepezil.

Results: There were significant improvements of RCPM in the total scores between one year donepezil treatment $(p=0.013)$, in both Set A score $(p=0.002)$ and Set $A B$ score $(p=0.015)$, but trend in the Set B score $(p=0.083)$.

Conclusion: Donepezil is useful for improving visuo-spatial impairment in DLB, but not for problem-solving impairment.
\end{abstract}

KEY WORDS: Lewy body dementia; Cholinesterase inhibitors; Raven's Colored Progressive Matrices; Visual spatial processing.

\section{INTRODUCTION}

Dementia with Lewy bodies (DLB) is the second most common neurocognitive disorder next to Alzheimer's disease $(\mathrm{AD}),{ }^{1)}$ and it is characterized by complex visual hallucinations, progressive cognitive dysfunction, and parkinsonism. ${ }^{2}$ Visual hallucination is one of the core features of DLB, present in $54 \%$ to $70 \%$ of DLB patients, and is one of the factors that increases caregiver burden. ${ }^{4)}$ It has been reported that the visual hallucinations of DLB are caused by visuo-spatial, visuo-perceptual, or attention deficits.

Cholinesterase inhibitors such as donepezil have been used to treat cognitive dysfunction and neuropsychiatric symptoms. ${ }^{6}$ Several reports have shown that donepezil improves the symptoms associated with progressive cognitive dysfunction. ${ }^{7,8)}$ In addition, imaging studies have reported that donepezil also improves visual hallucina-

Received: October 24, 2016/Revised: December 8, 2016

Accepted: December 20, 2016

Address for correspondence: Takaaki Mori, MD, PhD Department of Neuropsychiatry, Molecules and Function, Ehime University Graduate School of Medicine, Shitsukawa, Toon, Ehime 791-0295, Japan

Tel: +82-81-89-960-5315, Fax: +82-81-89-960-5317

E-mail: taka960mori@gmail.com

(c) This is an Open-Access article distributed under the terms of the Creative Commons Attribution Non-Commercial License (http://creativecommons.org/licenses/by-nc/4.0) which permits unrestricted non-commercial use, distribution, and reproduction in any medium, provided the original work is properly cited. tions. ${ }^{9,10)}$ On the other hand, it is well known that visuospatial deficits are worse in DLB than in $\mathrm{AD} .^{8,11,12)}$ Cholinergic dysfunction in DLB is reported to be related to visual responses in the visual cortex. ${ }^{13,14)}$

The non-verbal Raven's Colored Progressive Matrices (RCPM) test is a psychological tool used to assess both visual perception and reasoning ability. ${ }^{15)}$ The RCPM score is not affected by various physical disabilities such as aphasia, cerebral palsy, and deafness. ${ }^{16)}$ The RCPM score was reported to be lower in DLB than in AD in one observational study. ${ }^{17)}$

From these, visuo-spatial function of DLB is impaired and is possible to be improved by cholinesterase inhibitors. To the best of our knowledge, there are no longitudinal studies of how donepezil affects visuo-spatial deficits assessed by RCPM in DLB subjects. The present study examined how donepezil affects visuo-spatial function, as assessed by RCPM, in DLB subjects.

\section{METHODS}

\section{Subjects}

Untreated DLB patients ( $\mathrm{n}=21$; mean age, $78.7 \pm 4.5$ years) who were outpatients at Ehime University Hospital and Zaidan Niihama Hospital were enrolled. DLB sub- 
jects were diagnosed according to the diagnostic criteria for probable $\mathrm{DLB}^{2)}$ and were living with at least one caregiver. Some patients had psychiatric symptoms, but they could receive neuropsychological tests. Demographic data of DLB subjects are shown in Table 1. All patients were right-handed and had no cerebral vascular lesions on head computed tomography or magnetic resonance imaging (MRI). Patients who had a past history of psychiatric disorders and severe cardiovascular disease were excluded. All participants were of unrelated Japanese origin and signed written, informed consent forms approved by the institutional ethics committees of Ehime University Graduate School of Medicine and Zaidan Niihama Hospital.

\section{Evaluation of DLB Symptoms}

Cognitive impairments were evaluated by the MiniMental State Examination (MMSE). ${ }^{18)}$ The severity of dementia was assessed by the Clinical Dementia Rating

A

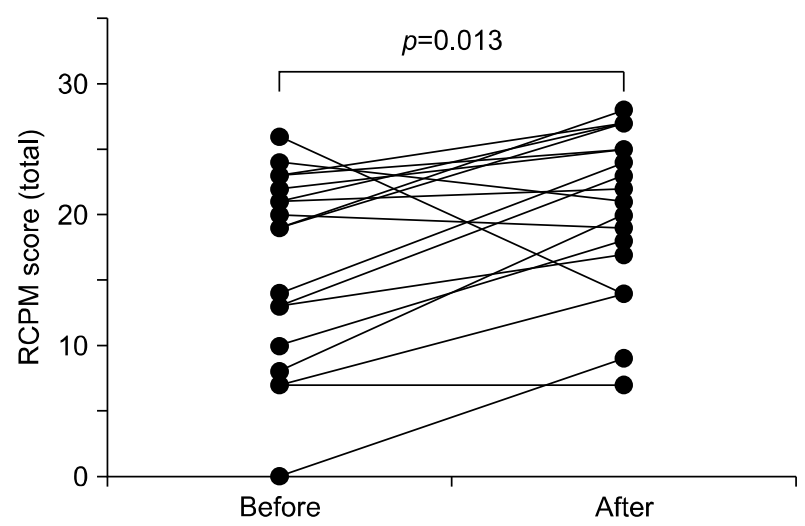

C
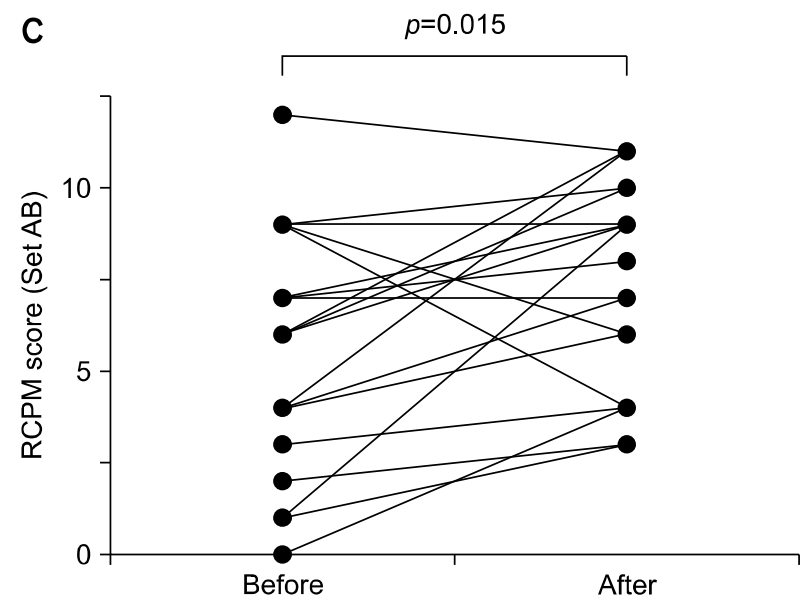

(CDR). Neuropsychiatric symptoms were evaluated by the neuropsychiatric inventory (NPI) assessed by caregivers. ${ }^{19)}$ We explained the method of RCPM to the participants and started the examination after they understood

Table 1. The demographic data and clinical data for DLB subjects

\begin{tabular}{lc}
\hline \multicolumn{1}{c}{ Characteristic } & DLB subject \\
\hline Subject $(n)$ & 21 \\
Sex $(n)$, male:female & $6: 15$ \\
Age $(y r)$ & $78.7 \pm 4.5$ \\
Age of onset (yr) & $77.0 \pm 4.6$ \\
Duration of illness $(y r)$ & $1.7 \pm 1.4$ \\
Education (yr) & $9.4 \pm 2.1$ \\
MMSE total score & $20.2 \pm 6.1$ \\
NPI-10 total score & $19.3 \pm 20.8$ \\
CDR score (0:0.5:1:2) & $1: 7: 10: 3$ \\
\hline
\end{tabular}

Values are presented as number only or mean \pm standard deviation. DLB, dementia with Lewy bodies; MMSE, Mini-Mental State Examination; NPI, neuropsychiatric inventory; CDR, Clinical Dementia Rating.

B

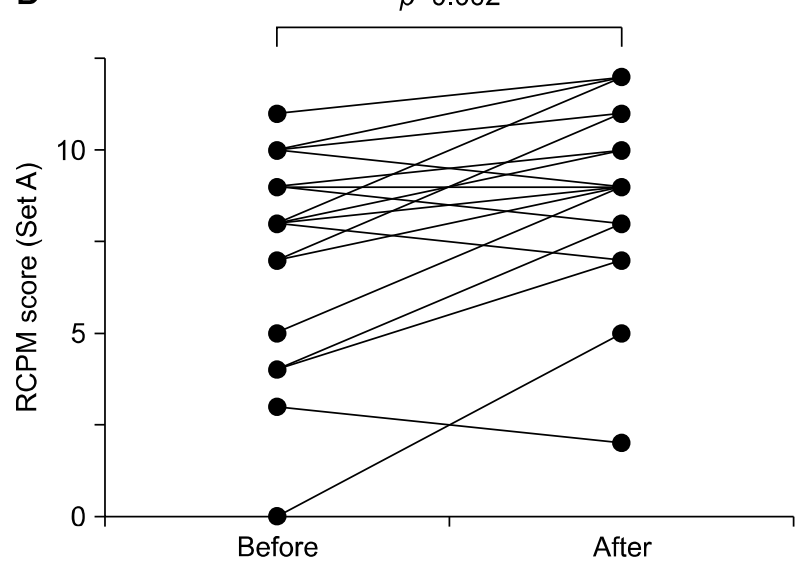

D

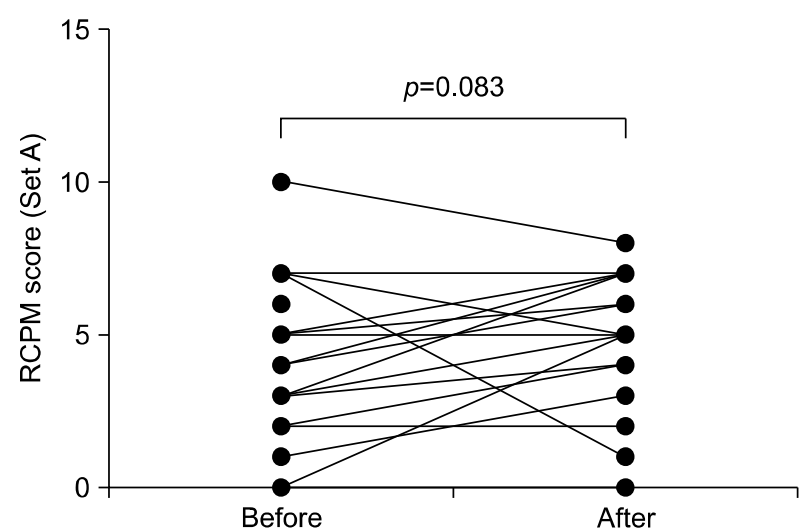

Fig. 1. The non-verbal Raven's Colored Progressive Matrices (RCPM) scores before and after taking donepezil. (A) Total score, (B) Set A score, (C) Set AB score, and (D) Set B score. 
and concentrated it. The visuospatial deficit was evaluated by the RCPM. ${ }^{15)}$ These clinical data are also shown in Table 1.

\section{Study Protocol}

MMSE, CDR, NPI, and RCPM assessments were performed at the time of starting donepezil, and post prescribing RCPM was evaluated within one year after starting donepezil.

\section{Statistical Analysis}

Statistical analyses were performed using IBM SPSS Statistics ver 22.0 software (IBM Japan, Tokyo, Japan). Comparisons of pre and post RCPM scores (total, Set A, $\mathrm{AB}$, and $\mathrm{B}$ ) were conducted by the Wilcoxon signed-rank test. Linear regression analyses were performed to ascertain whether age, age of onset, educational years, duration of illness, dose of donepezil, mean administration period (week), change of drugs without donepezil between pre and post RCPM test, MMSE, improved MMSE score, NPI, and CDR were associated with improved RCPM scores (total, Set A, AB, and B). Statistical significance was defined at the $95 \%$ level $(p=0.05)$.

\section{RESULTS}

\section{Comparisons between Pre- and Post-RCPM Scores}

There were significant improvements in the RCPM total score ( $p=0.013$, Fig. 1A), Set $\mathrm{A}(p=0.002$, Fig. 1B $)$, and Set $\mathrm{AB}$ ( $p=0.015$, Fig. $1 \mathrm{C})$, but not in Set $\mathrm{B}(p=0.083$, Fig. 1D). The details of the RCPM scores are shown in Table 2. The cut-off score of total RCPM score is 24/25 in Japanese version. ${ }^{20)}$ According to this cut-off score, most patients had visuo-spatial dysfunction when pre RCPM test was conducted. The mean donepezil dose was $5.1 \pm 1.8 \mathrm{mg}$, and the mean administration period was $21.1 \pm 12.4$ weeks. Some DLB subjects took other medications in pre-test (antipsychotics, one; antidepressants, two) and post-test

Table 2. The change of non-verbal Raven's Colored Progressive Matrices scores and Mini-Mental State Examination (MMSE)

\begin{tabular}{lrrc}
\hline Characteristic & Before (A) & After (B) & $\begin{array}{c}\text { Improved } \\
\text { score (B-A) }\end{array}$ \\
\hline Total $(n=21)$ & $17.1 \pm 7.8$ & $21.3 \pm 6.5$ & $4.2 \pm 5.8$ \\
Set $A(n=21)$ & $7.4 \pm 2.8$ & $9.0 \pm 2.4$ & $1.6 \pm 2.0$ \\
Set AB $(n=21)$ & $5.8 \pm 3.3$ & $7.5 \pm 2.7$ & $1.8 \pm 3.1$ \\
Set $B(n=21)$ & $4.0 \pm 2.6$ & $4.8 \pm 2.1$ & $0.9 \pm 2.4$ \\
MMSE $(n=16)$ & $19.7 \pm 6.4$ & $21.9 \pm 6.2$ & $2.2 \pm 3.7$ \\
\hline
\end{tabular}

Values are presented as mean \pm standard deviation. (antipsychotics, three; antidepressants, two; antiparkinson drugs, two).

\section{Linear Regression Analyses}

Age, age of onset, educational years, duration of illness, dose of donepezil, mean administration period, change of drugs without donepezil between pre and post RCPM test, MMSE, improved MMSE score, NPI, and CDR were not significantly associated with improved RCPM scores for total, Set A, Set AB, and Set B scores.

\section{DISCUSSION}

The results of the present study show that donepezil may improve RCPM scores in DLB subjects. Interestingly, Set $A$ and Set $A B$ scores were significantly improved, but not Set B scores. From linear regression analysis, the improvement was not associated with age, age of onset, duration of illness, dose of donepezil, MMSE, NPI, or CDR.

Let us first examine the improvements of the RCPM scores in detail. In the previous study, identity, similarity, and difference in Set A constitute the main relational features of the elements. In Set AB, spatial orientation such as symmetry or location is the predominant theme, while both logical and spatial principles in Set B are involved in figure construction. ${ }^{21)}$ It appears that donepezil improves mainly spatial orientation involved with identity, similarity, and difference, which constitute the main relational features of elements in DLB subjects. However, logical thinking about spatial features was not improved by donepezil treatment. According to the study that divided the RCPM elements into visuo-spatial and problem-solving impairment, ${ }^{22)}$ visuo-spatial impairment was improved, but problem-solving impairment was not improved by donepezil in the present study. Actually, Saumier et al. ${ }^{23)}$ reported that the domains of visual-spatial motor abilities were improved by donepezil in AD subjects.

Second, let us examine the improvement of visuo-spatial function by donepezil. Anatomically, posterior cortical atrophy leads to visual-spatial dysfunction. A rodent study suggested that the pharmacological activation of acetylcholine receptors relates to the visual response. ${ }^{24)}$ Cerami et al. ${ }^{25)}$ using $\left[{ }^{18} \mathrm{~F}\right]$ FDG-positron emission tomography (PET) and diffusion tensor imaging-MRI, reported that the occipital-parietal-frontal network could be at the basis of visuo-perceptual and visuo-spatial integration. It was reported that DLB is associated with cholinergic dysfunction on PET using radiolabeled acetylcholine analogues. ${ }^{26)}$ In that study, the deficit was in the posterior 
cortical regions, the inferior temporal gyrus, the supramarginal gyrus, and the posterior cingulate. Tiraboschi et $a l{ }^{27)}$ reported that cholinergic dysfunction is more marked in DLB than in AD. In addition, cerebral blood flow in the occipital cortex is increased by donepezil. ${ }^{9)}$ From these studies, we assumed that visuo-spatial function was improved through the increased cerebral blood flow in the occipital cortex with donepezil treatment.

This study had several limitations. One limitation is that there may have been Type II error due to the small sample size. In addition, the effect of other medications could not be considered sufficiently due to small sample size. Second is that cognitive fluctuation of DLB was not assessed when conducting the RCPM test. Cognitive fluctuation is a well-known symptom in DLB, and it also has a significant effect on cognitive function and activities of daily living in DLB patients and causes an increased care burden for caregivers. ${ }^{28)}$ Cognitive fluctuation may have affected the RCPM scores in the present study. In future studies, RCPM scores should be evaluated at the same time as cognitive fluctuation in a large sample. Third is that we did not recruit the control arm without donepezil. In future study, it is need to evaluate the improvement of RCPM scores with donepezil compared to control subjects without donepezil. Lastly, Ikeda et $a{ }^{29)}$ reported that MMSE score significantly improved compared to placebo in the 10-mg group, but not in 5-mg group. We had to increase the dose of donepezil because of the evidence of Ikeda $e t a l .^{29)}$ Even with low dose, the subjects showed significant improvement with donepezil treatment. The donepezil dose of DLB patients in this study is $5.1 \pm 1.8$. It is possible that higher dose of donepezil improve visuospatial function.

\section{acknowledgments}

The authors would like to thank the patients and family members who participated in this study and the staff of Zaidan Niihama Hospital, Ehime, Japan for understanding the purpose of this study. This work was partially supported by a Health and Labor Science Research Grant from the Japanese Ministry of Health, Labor and Welfare, a Grant-in-Aid for Scientific Research from the Japanese Ministry of Education, Culture, Sports, Science and Technology, and JSPS KAKENHI Grant Number 25861015.

\section{REFERENCES}

1. McKeith IG, Galasko D, Kosaka K, Perry EK, Dickson DW, Hansen LA, et al. Consensus guidelines for the clinical and pathologic diagnosis of dementia with Lewy bodies (DLB): report of the consortium on DLB international workshop.
Neurology 1996;47:1113-1124.

2. McKeith IG, Dickson DW, Lowe J, Emre M, O’Brien JT, Feldman $\mathrm{H}$, et al. Diagnosis and management of dementia with Lewy bodies: third report of the DLB Consortium. Neurology 2005;65:1863-1872.

3. Aarsland D, Ballard C, Larsen JP, McKeith I. A comparative study of psychiatric symptoms in dementia with Lewy bodies and Parkinson's disease with and without dementia. Int $J$ Geriatr Psychiatry 2001;16:528-536.

4. Galvin JE, Duda JE, Kaufer DI, Lippa CF, Taylor A, Zarit SH. Lewy body dementia: caregiver burden and unmet needs. Alzheimer Dis Assoc Disord 2010;24:177-181.

5. Mori E, Ikeda M, Kosaka K; Donepezil-DLB Study Investigators. Donepezil for dementia with Lewy bodies: a randomized, placebo-controlled trial. Ann Neurol 2012;72:41-52.

6. Matsunaga S, Kishi T, Yasue I, Iwata N. Cholinesterase inhibitors for Lewy body disorders: a meta-analysis. Int $J$ Neuropsychopharmacol 2015;19:pyv086.

7. Thomas AJ, Burn DJ, Rowan EN, Littlewood E, Newby J, Cousins D, et al. A comparison of the efficacy of donepezil in Parkinson's disease with dementia and dementia with Lewy bodies. Int J Geriatr Psychiatry 2005;20:938-944.

8. Mori E, Shimomura T, Fujimori M, Hirono N, Imamura T, Hashimoto M, et al. Visuoperceptual impairment in dementia with Lewy bodies. Arch Neurol 2000;57:489-493.

9. Mori T, Ikeda M, Fukuhara R, Nestor PJ, Tanabe H. Correlation of visual hallucinations with occipital $r C B F$ changes by donepezil in DLB. Neurology 2006;66:935-937.

10. Satoh M, Ishikawa H, Meguro K, Kasuya M, Ishii H, Yamaguchi S. Improved visual hallucination by donepezil and occipital glucose metabolism in dementia with Lewy bodies: the Osaki-Tajiri project. Eur Neurol 2010;64:337-344.

11. Ala TA, Hughes LF, Kyrouac GA, Ghobrial MW, Elble RJ. Pentagon copying is more impaired in dementia with Lewy bodies than in Alzheimer's disease. J Neurol Neurosurg Psychiatry 2001;70:483-488.

12. Collerton D, Burn D, McKeith I, O'Brien J. Systematic review and meta-analysis show that dementia with Lewy bodies is a visual-perceptual and attentional-executive dementia. Dement Geriatr Cogn Disord 2003;16:229-237.

13. Kimura F, Fukuda M, Tsumoto T. Acetylcholine suppresses the spread of excitation in the visual cortex revealed by optical recording: possible differential effect depending on the source of input. Eur J Neurosci 1999;11:3597-3609.

14. Silver MA, Shenhav A, D'Esposito M. Cholinergic enhancement reduces spatial spread of visual responses in human early visual cortex. Neuron 2008;60:904-914.

15. Raven JC. Colored progressive matrices sets $A, A B, B$. London:H.K. Lewis; 1947.

16. Spreen O, Staruss E. A compendium of neuropsychological tests: administration, norms, and commentary. 2nd ed. Oxford:Oxford University Press; 1998.

17. Kawai Y, Miura R, Tsujimoto M, Sakurai T, Yamaoka A, Takeda A, et al. Neuropsychological differentiation between Alzheimer's disease and dementia with Lewy bodies in a memory clinic. Psychogeriatrics 2013;13:157-163.

18. Folstein MF, Folstein SE, McHugh PR. "Mini-mental state". A practical method for grading the cognitive state of patients for the clinician. J Psychiatr Res 1975;12:189-198.

19. Cummings JL, Mega M, Gray K, Rosenberg-Thompson S, Carusi DA, Gornbein J. The Neuropsychiatric Inventory: comprehensive assessment of psychopathology in dementia. Neurology 1994;44:2308-2314.

20. Sugishita M, Yamazaki K. Raven's colored progressive matrices (in Japanese). Tokyo:Nihon Bunka Kagakusya;1993. 
21. Weichbold V, Herka H. Performance of hearing impaired children on Raven's Coloured Progressive Matrices Test. Int J Pediatr Otorhinolaryngol 2003;67:1213-1217.

22. Ambra FI, Iavarone A, Ronga B, Chieffi S, Carnevale G, Iaccarino L, et al. Qualitative patterns at Raven's colored progressive matrices in mild cognitive impairment and Alzheimer's disease. Aging Clin Exp Res 2016;28:561-565.

23. Saumier D, Murtha S, Bergman H, Phillips N, Whitehead $\mathrm{V}$, Chertkow H. Cognitive predictors of donepezil therapy response in Alzheimer disease. Dement Geriatr Cogn Disord 2007;24:28-35.

24. Soma S, Shimegi S, Suematsu N, Sato H. Cholinergic modulation of response gain in the rat primary visual cortex. Sci Rep 2013;3:1138.

25. Cerami C, Crespi C, Della Rosa PA, Dodich A, Marcone A, Magnani G, et al. Brain changes within the visuo-spatial attentional network in posterior cortical atrophy. J Alzheimers
Dis 2015;43:385-395.

26. Shimada H, Hirano S, Shinotoh H, Aotsuka A, Sato K, Tanaka N, et al. Mapping of brain acetylcholinesterase alterations in Lewy body disease by PET. Neurology 2009; 73:273-278.

27. Tiraboschi P, Hansen LA, Alford M, Merdes A, Masliah E, Thal LJ, et al. Early and widespread cholinergic losses differentiate dementia with Lewy bodies from Alzheimer disease. Arch Gen Psychiatry 2002;59:946-951.

28. Lee DR, Taylor JP, Thomas AJ. Assessment of cognitive fluctuation in dementia: a systematic review of the literature. Int J Geriatr Psychiatry 2012;27:989-998.

29. Ikeda M, Mori E, Matsuo K, Nakagawa M, Kosaka K. Donepezil for dementia with Lewy bodies: a randomized, placebo-controlled, confirmatory phase III trial. Alzheimers Res Ther 2015;7:4. 